(2) Open Access Full Text Article

REVIEW

\title{
Dronedarone: current evidence for its safety and efficacy in the management of atrial fibrillation
}

This article was published in the following Dove Press journal:

Drug Design, Development and Therapy

31 December 2010

Number of times this article has been viewed

\section{Patrick A Schweizer \\ Rüdiger Becker \\ Hugo A Katus \\ Dierk Thomas \\ Department of Cardiology, Medical University Hospital, Heidelberg, Germany}

\begin{abstract}
Atrial fibrillation (AF) is the most common sustained arrhythmia. Management of AF includes rate control, rhythm control if necessary, prevention of thromboembolic events, and treatment of the underlying disease. Rate control is usually achieved by pharmacological suppression of calcium currents or by applying $\beta$-blockers or digitalis compounds. In contrast, the number of compounds available for rhythm control is still limited. Class Ic agents increase mortality in patients with structural heart disease, and amiodarone harbors an extensive side effect profile despite its efficacy in maintaining sinus rhythm. Furthermore, rhythm control by these compounds has not been shown to reduce patient mortality. Dronedarone is a new antiarrhythmic drug that has been developed to provide rhythm and rate control in AF patients with fewer side effects compared with amiodarone. This review primarily focuses on clinical trials evaluating efficacy and safety of the novel drug. Conclusions from these studies are critically reviewed, and recommendations for clinical practice are discussed. Dronedarone significantly reduced the incidence of hospitalization due to cardiovascular events or death in high-risk patients with atrial fibrillation (ATHENA trial). However, dronedarone was less efficient than amiodarone in maintaining normal sinus rhythm (DIONYSOS trial) and is contraindicated in severe or deteriorating heart failure (ANDROMEDA trial). In summary, dronedarone represents a valuable addition to the limited spectrum of antiarrhythmic drugs and is currently recommended in patients with paroxysmal and persistent AF to achieve rate and rhythm control, excluding cases of severe or unstable congestive heart failure.
\end{abstract}

Keywords: dronedarone, atrial fibrillation, antiarrhythmic therapy

\section{Search strategy}

We searched Medline, PubMed, BIOSIS, and Cochrane Library databases for original articles, reviews, comments, and meta-analyses. Search terms included: "dronedarone", "atrial fibrillation", "rhythm control", "rate control", "antiarrhythmic therapy", and "ion-channel blocker". The search focused on publications from the past 5 years but did not exclude older publications of general interest. We also searched the reference lists of articles identified by this search strategy and selected those we judged relevant. Date of last search was 14 November, 2010.

\section{Introduction}

Atrial fibrillation (AF) is the most common sustained arrhythmia and worsens the overall prognosis of patients with cardiovascular diseases by significantly increasing the risk of serious cardiovascular complications (eg, heart failure, stroke, cardiovascular hospitalization and death). ${ }^{1-6} \mathrm{AF}$ is associated with multiple cardiovascular conditions 
including arterial hypertension, dyslipidemia, heart failure, valvular heart disease, coronary artery disease, and diabetes mellitus. $^{7,8}$ The disease has a current prevalence of $0.95 \%$ in the United States and is strictly age dependent: $0.1 \%$ of all individuals under 55 years, but up to $9.0 \%$ of those over 80 years are affected. ${ }^{9}$ Interestingly, in a projected calculation AF prevalence was predicted to increase by $\geq 2.5$-fold by the year $2050 .^{10,11}$

Sustained rapid ectopic activity and reentry represent mechanisms that contribute to the development of AF. ${ }^{12-14}$ Furthermore, atrial remodeling is an important determinant that leads to generation and maintenance of these factors. ${ }^{15}$ According to the leading circle theory, reentry circuits are favored by short refractoriness and slow conduction. ${ }^{16,17}$ The hypothesis of spiral-wave activity proposes a circulating wave front that rapidly rotates around a central core, depending on tissue excitability and refractoriness as well as on central core stability. ${ }^{17-19}$ Consistent with this theory, conditions that increase excitability and shorten refractoriness promote AF maintenance by enhancing spiral-wave rotation and central core stability. Drugs aiming at suppression of AF target these mechanisms. Classic antiarrhythmic $\mathrm{Na}^{+}$-current inhibitors (class I drugs) decrease excitability and destabilize rotator activity, while $\mathrm{K}^{+}$-channel blocking (or class III) drugs suppress reentry mechanisms by extending action potential duration and repolarization. ${ }^{20}$

Traditionally, AF management focuses on stroke prevention and symptom management. Preventing thromboembolic complications is a major priority and reduces morbidity and mortality, and trials of oral anticoagulant therapy demonstrated a substantial risk reduction. ${ }^{21}$ During AF, normal atrioventricular node conduction can lead to rapid ventricular rate response, resulting in impairment of left ventricular function and severe limitation of physical activity, which is a frequent cause for patient hospitalization. Rate control is usually achieved by pharmacological suppression of calcium currents or by applying $\beta$-blockers or digitalis compounds until heart rate is decreased to a less symptomatic state. In contrast, rhythm control aims to convert the patient's heart rhythm to normal sinus rhythm (NSR) and to maintain NSR once achieved. Recent studies comparing rate and rhythm control strategies (ie, combined results of PIAF [Pharmacological Intervention in Atrial Fibrillation], STAF [The Strategies of Treatment of Atrial Fibrillation], and AFFIRM [Atrial Fibrillation Follow-up Investigation of Rhythm Management]) showed that neither achieved proven morbidity/mortality benefits, while rhythm control using the class III drug amiodarone was associated with higher hospitalization rates. ${ }^{22-25}$ On the other hand, class I antiarrhythmic agents have been shown to increase mortality in patients with structural heart disease. ${ }^{26}$ Consequently American College of Cardiology (ACC)/American Heart Association (AHA) 2006, and European Society of Cardiology (ESC) 2010 guidelines for the management of patients with AF recommended rate control as first-line therapy. ${ }^{7,8,22}$ If the patient remains symptomatic, therapy should be continued or supplemented by an appropriate rhythm control drug. Amiodarone is the most frequently used antiarrhythmic drug to achieve and maintain NSR. ${ }^{23}$ According to the ACC/AHA 2006 and ESC 2010 guidelines, amiodarone should be administered at a maintenance dose of 200 to $400 \mathrm{mg} /$ day. $^{7,8,27}$ In addition, amiodarone has a heart rate lowering effect and can be used simultaneously for rate control, particularly if classic rate control agents failed or are contraindicated. ${ }^{7,8}$ However, the use of amiodarone is limited by significant adverse effects including decreased blood pressure, pulmonary toxicity, skin discoloration, thyroid toxicity, corneal deposits, optic neuropathy, and sinus bradycardia. As a result, many patients are not eligible or refuse to take the drug. Consequently, pharmaceutical research has focused on developing more favorable multichannel-blocking agents as well as novel ion-channel and nonchannel targets. ${ }^{20,28}$

Dronedarone was approved in July 2009 and is indicated for the treatment of adults with nonpermanent AF to prevent recurrence, or to lower ventricular rate. It is a modified derivate of amiodarone and its development targeted on finding a substance combining beneficial antiarrhythmic effects without the potential adverse events of amiodarone.

\section{Aims}

This review focuses on pharmacological characteristics and clinical trial series performed to test efficacy and safety of dronedarone in the treatment of atrial fibrillation. Furthermore, morbidity/mortality endpoints evaluated in the largest antiarrhythmic drug trial to date, ATHENA (Prevention of Cardiovascular Hospitalization or Death from Any Cause in Patients with Atrial Fibrillation/Atrial Flutter) are critically discussed with respect to current evidence.

\section{Pharmacology Chemistry ${ }^{29}$}

Dronedarone is a benzofuran molecule, which is chemically related to amiodarone. Unlike amiodarone, it does not harbor the iodine moieties causing thyroid problems. Moreover the addition of a methyl sulfonyl group decreases its lipophilicity 
and shortens its plasma half-life which is thought to reduce organ toxicity due to accumulative effects (see Figure 1).

\section{Mechanistic properties}

Similar to amiodarone, dronedarone is a multichannel blocker that meets criteria of all four Vaughan Williams antiarrhythmic drug classes: rate-dependent inhibition of the rapid $\mathrm{Na}^{+}$current (class I), $\alpha-$ and $\beta$-adrenergic receptor inhibition (class II), blockade of $\mathrm{K}^{+}$outward currents as the main mechanism of action (class III), and blockade of slow $\mathrm{Ca}^{2+}$ inward currents (class IV). ${ }^{30,31}$ Class I and III effects increase refractory periods and decelerate cardiac conduction, providing mechanisms that induce rhythm control. Although precise mechanisms of dronedarone action are not fully understood, balanced inhibition of multiple outward currents may explain the decrease in the transmural dispersion of repolarization, which prevents significant proarrhythmic effects. ${ }^{32}$ Furthermore, in contrast to pure $\mathrm{I}_{\mathrm{K}}$ dronedarone increases action potential duration and effective refractory period without reverse use-dependency, preventing the risk of early afterdepolarization..$^{32,33}$ In addition, class II and IV effects contribute to rate control properties as well as the anti-adrenergic (class II) and blood pressure lowering (class IV) effects of the drug. ${ }^{34,35}$ The direct vasodilatatory properties of both amiodarone and dronedarone have also been examined. ${ }^{36}$

\section{Pharmacokinetics/pharmacodynamics ${ }^{29}$}

\section{Absorption}

Dronedarone is well absorbed after oral administration $(>70 \%)$. Because of pre-systemic first-pass metabolism, the absolute bioavailability is $15 \%$ when administered with food, as recommended, increasing dronedarone bioavailability by

\section{A}

\section{Dronedarone}

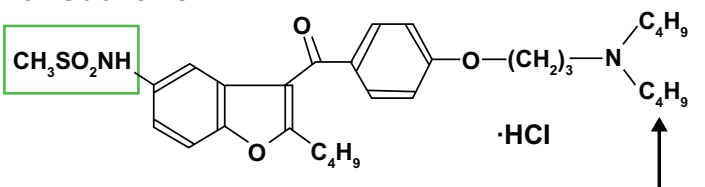

B

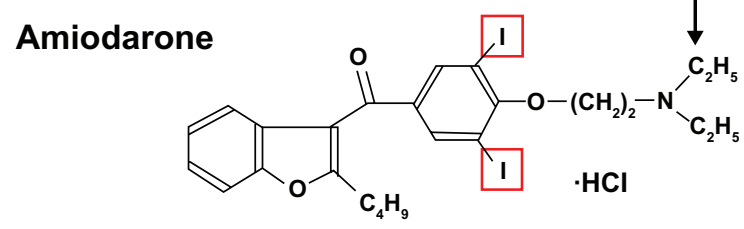

Figure I Chemical structure of dronedarone A). The substance is based on a benzofuran molecule and does not possess the iodine moieties (red frame) causing thyroid problems associated with amiodarone $\mathbf{B}$ ). The addition of a methyl sulfonyl group (green frame) decreases its lipophilicity and shortens its plasma half-life to reduce organ toxicity.
2- to 4-fold, due to significant first pass metabolism. After oral administration, peak plasma concentrations of dronedarone and the main circulating active metabolite (N-debutyl metabolite) are reached within 3 to 6 hours. Under $400 \mathrm{mg}$ twice a day, steady state is reached within 4 to 8 days of treatment.

\section{Distribution}

The in vitro plasma protein binding of dronedarone and its $\mathrm{N}$-debutyl metabolite is $99.7 \%$ and $98.5 \%$, respectively, and is not capable of saturation. Both compounds bind mainly to albumin.

\section{Metabolism}

Like amiodarone, dronedarone is metabolized mainly by CYP3A4. It forms the predominant circulating metabolite by N-debutylation followed by oxidation and oxidative deamination. The N-debutyl metabolite exhibits pharmacodynamic activity, but is 3 to 10 times less potent than dronedarone. After oral administration, approximately $6 \%$ of the labeled dose is excreted in urine and $84 \%$ is excreted in feces, both mainly as metabolites. The terminal elimination half-life of dronedarone is $\sim 25$ to 30 hours and that of its N-debutyl metabolite $\sim 20$ to 25 hours. Dronedarone and its metabolite applied in a $400 \mathrm{mg}$ twice a day treatment doses are eliminated from plasma within 2 weeks after therapy stops. Compared with amiodarone, dronedarone has less active metabolites and has quicker onset and offset of actions and shorter half-life as well as a smaller volume of distribution.

\section{Pharmacodynamics}

Electrocardiographic changes include a dose-related decrease of heart rate at rest and under exercise, and moderate increase of PR- and QTc-intervals. In addition moderate lowering of blood pressure was observed. ${ }^{37}$ Furthermore an increase of serum creatinine levels by about $0.1 \mathrm{mg} / \mathrm{dL}$ was detected, reaching plateau after 7 days of treatment and being reversible after discontinuation of the drug. A similar effect has been demonstrated with amiodarone. ${ }^{38}$ These effects have been shown to result from an inhibition of tubular creatinine secretion without affecting glomerular filtration rate. ${ }^{39}$ However, serum creatinine levels should be checked 1 week after onset of drug administration.

\section{Pharmacokinetic variability and pharmacodynamic interactions}

The main sources of pharmacokinetic variability identified are age, gender, and weight, but they all have only modest 
effects. Furthermore congestive heart failure, renal function, and even severe renal impairment do not significantly influence pharmacokinetics of dronedarone, and thus no dose adjustment is required. Moderate hepatic impairment alters pharmacokinetics by a 1.3-fold increase of steady-state dronedarone exposure, while active metabolite exposure decreased by 1.6- to 1.9-fold. Concomitant pharmacodynamic changes in ECG parameters were not detected. No dosage adjustment is recommended for moderate hepatic impairment, but dronedarone is contraindicated in severe hepatic failure. ${ }^{29}$

The primary extrinsic factors that influence pharmacokinetics of dronedarone are modulators of CYP3A4. The use of strong CYP3A inhibitors (eg, ketoconazole) together with dronedarone is contraindicated, and grapefruit juice should also be avoided.

Dronedarone can significantly increase statin levels by 2- to 4-fold and clinical signs of muscular toxicity should be monitored. Notably, pharmacodynamic interactions can be expected with $\beta$-blockers, calcium channel blockers and digoxin. Drugs that prolong the QT interval, potentially inducing torsade de pointes (TdP) tachykardia, such as phenothiazines, cisapride, bepridil, tricyclic antidepressants, oral macrolides and class I and III antiarrhythmic drugs are contraindicated. Only mild interaction was observed between dronedarone and warfarin with no clinically significant increase in international normalized ratio (INR). Pregnancy and nursing are both considered contraindications to dronedarone. It may cause fetal harm and thus should be avoided during pregnancy or in those who may become pregnant. Furthermore dronedarone may pose potential harm in nursing infants. The safety and efficacy of dronedarone have not been proven in children below the age of 18 years.

\section{Clinical trials DAFNE $^{37}$}

The DAFNE (Dronedarone Atrial Fibrillation study after Electrical Cardioversion) trial was designed as a phase II trial to establish the appropriate dose of dronedarone for preventing recurrence of AF after cardioversion in patients with persistent AF. DAFNE was a multinational, multicenter, prospective double-blind, placebo-controlled trial. Eligible patients showing persistent AF for $\geq 72$ hours and $<12$ months duration were randomized to receive either placebo or dronedarone tablets $400 \mathrm{mg}$ twice a day, $600 \mathrm{mg}$ twice a day or $800 \mathrm{mg}$ twice a day for a planned duration of 6 months. Patients who did not convert to NSR within 5 days after onset of medication were treated by electrical cardioversion. The primary endpoint included the time to first AF recurrence. Secondary endpoints were defined as spontaneous conversion of AF following randomization, heart rate in case of AF recurrence, and incidence of side effects. Patients included totaled 270, and 50 active centers in 11 countries contributed to the study.

Dronedarone $400 \mathrm{mg}$ twice a day significantly increased the time to first recurrence of AF compared with placebo. The $400 \mathrm{mg}$ twice a day dose showed best results in terms of efficacy and safety. Gastrointestinal (GI) side effects were the most frequent adverse event in the dronedarone groups. Although rare in the $400 \mathrm{mg}$ twice a day group $(1.3 \%$ of patients discontinued because of GI problems), patients in the $800 \mathrm{mg}$ twice a day group frequently reported GI problems and showed significant QT prolongation. Furthermore DAFNE demonstrated a significant lowering of ventricular rate of patients who relapsed into AF. In conclusion DAFNE identified $400 \mathrm{mg}$ twice a day as the best dosage of dronedarone for further testing in phase III trials.

\section{EURIDIS/ADONIS ${ }^{40}$}

EURIDIS (European Trial in Atrial Fibrillation or Flutter Patients Receiving Dronedarone for the Maintenance of Sinus Rhythm) and ADONIS (American-Australian Trial with Dronedarone in Atrial Fibrillation of Flutter Patients for the Maintenance of Sinus Rhythm) were initiated to assess the efficacy of dronedarone for maintaining NSR after electrical, pharmacological or spontaneous conversion from paroxysmal or persistent AF or atrial flutter (AFL). The studies were designed as double-blind, parallel-arm, placebo-controlled phase III trials and randomized 612 and 625 patients, respectively. Inclusion criteria included more than one AF episode within the previous 3 months. Patients with NYHA (New York Heart Association) class III or IV were excluded. The primary endpoint was the time to first recurrence of AF/ AFL. Secondary endpoints included symptomatic AF/AFL among adjudicated first AF/AFL recurrence, time between steady state and adjudicated first AF/AFL recurrence, mean ventricular rate during first AF recurrence, and cardiovascular hospitalization. Some patients in both arms received basal therapy for rate-control ( $\beta$-blocker, calcium-channel blocker, digoxin) and additional cardiovascular medication. In conclusion, dronedarone was superior to placebo in preventing recurrence of AF with $64.1 \%$ of patients having AF recurrence at 12 months compared with $75.2 \%$ in the placebo group $(P<0.001)$. Moreover dronedarone was also effective in controlling ventricular rates after AF relapse, as ventricular rates were $14 \mathrm{bpm}$ slower than in the placebo 


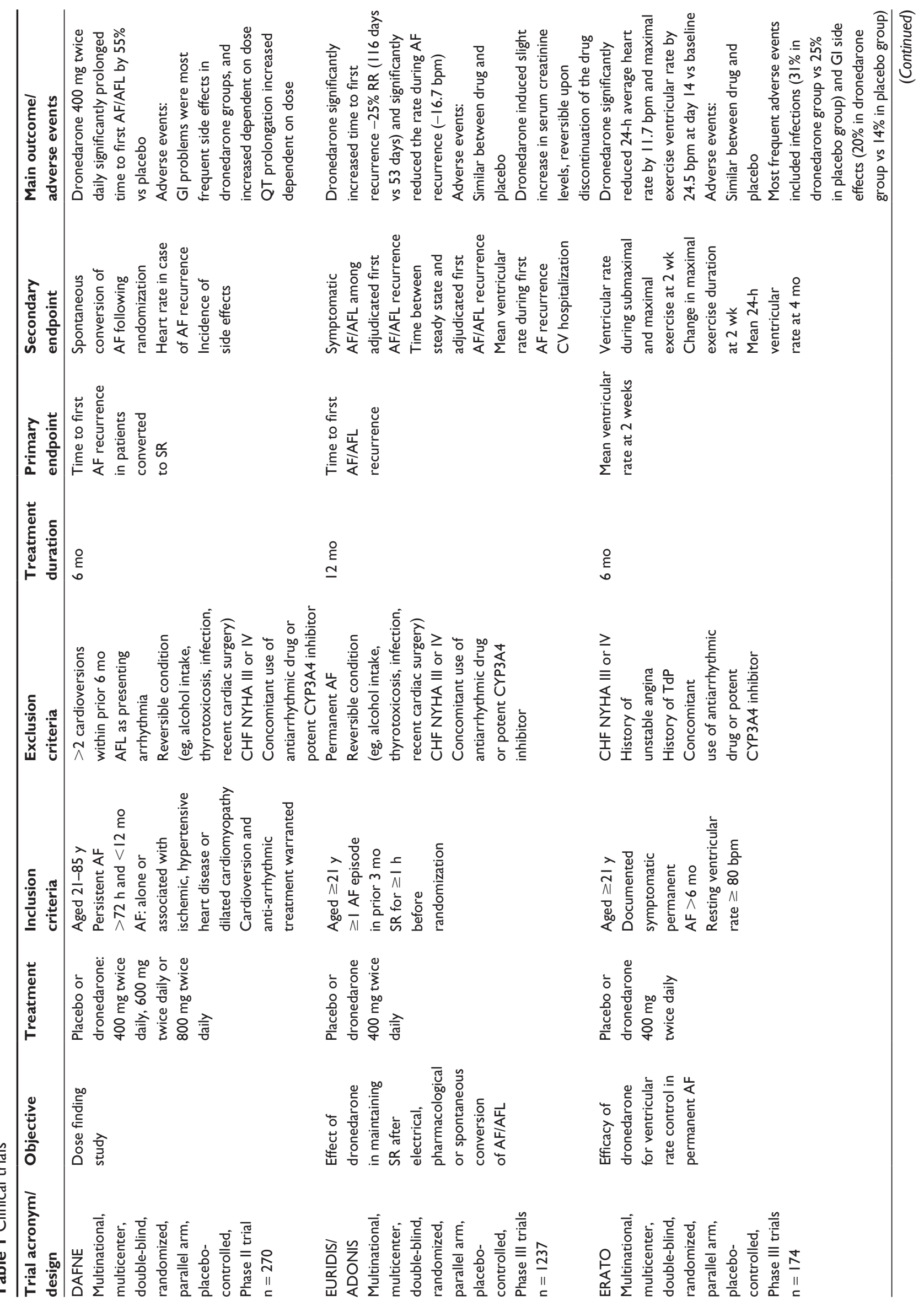




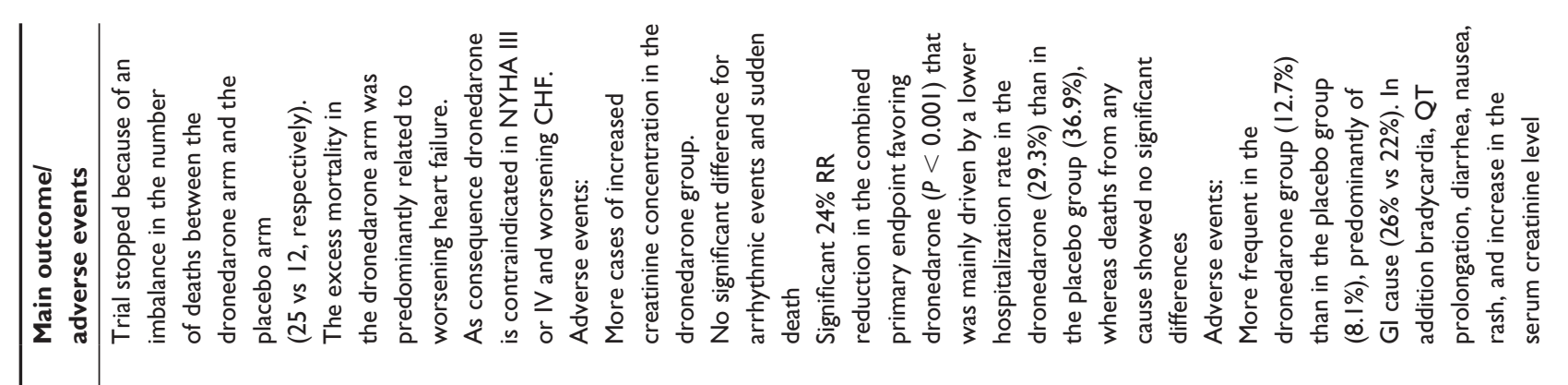

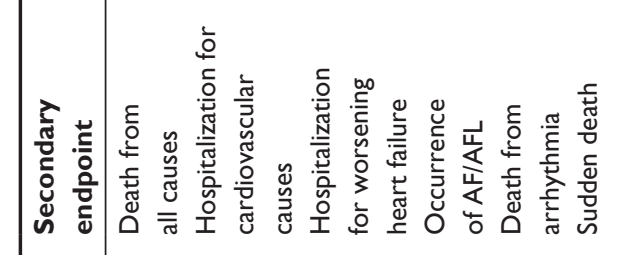

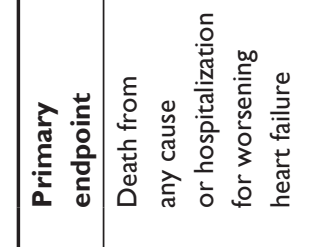

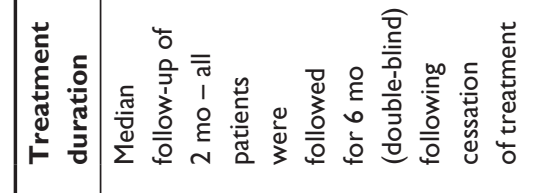
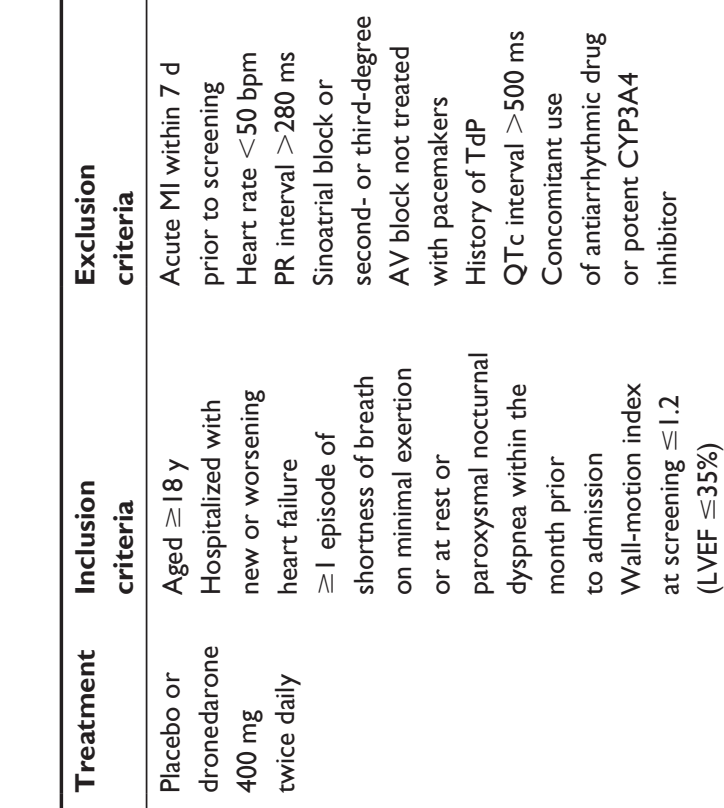

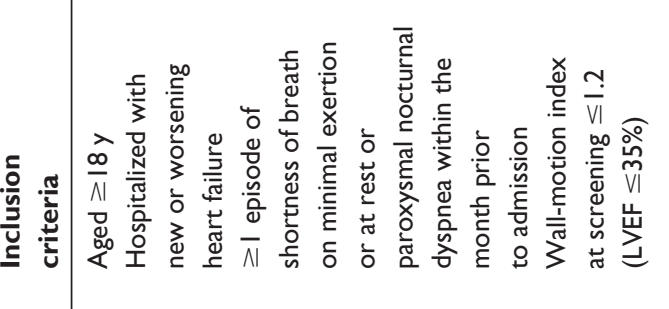

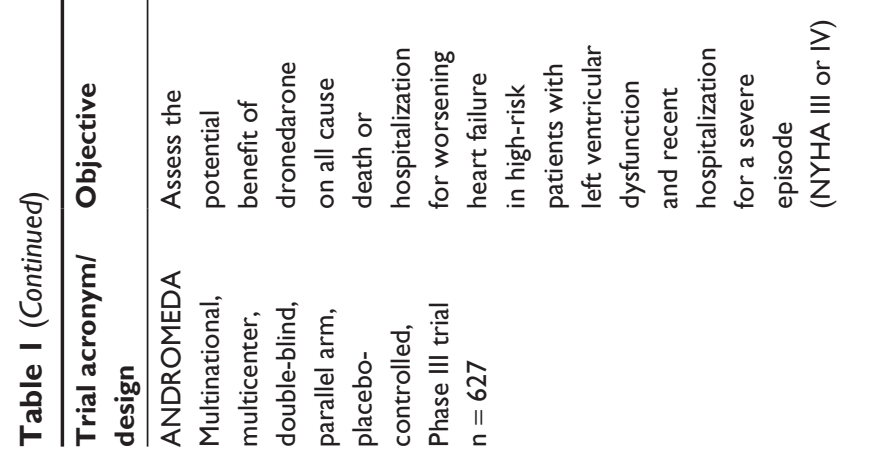

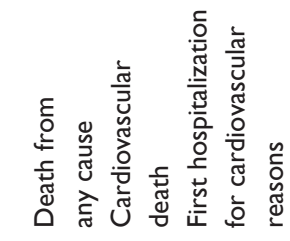

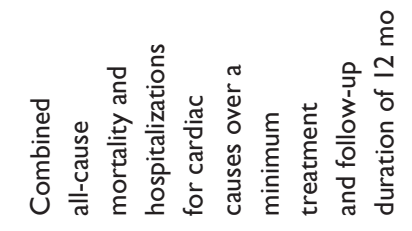

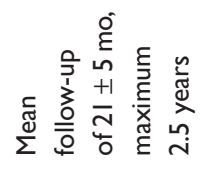

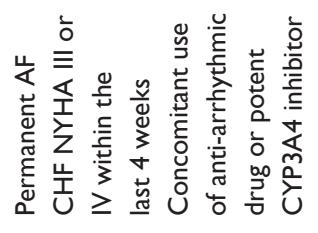

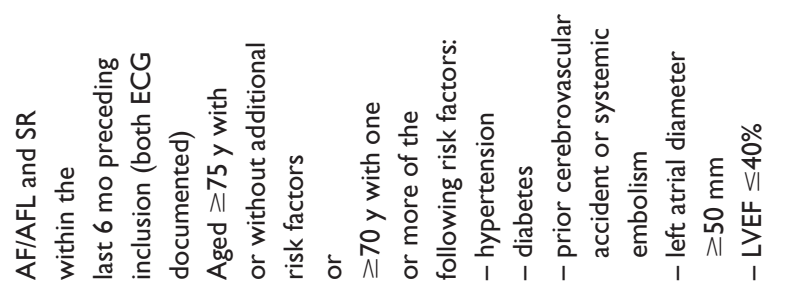

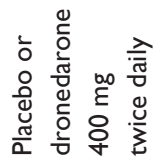

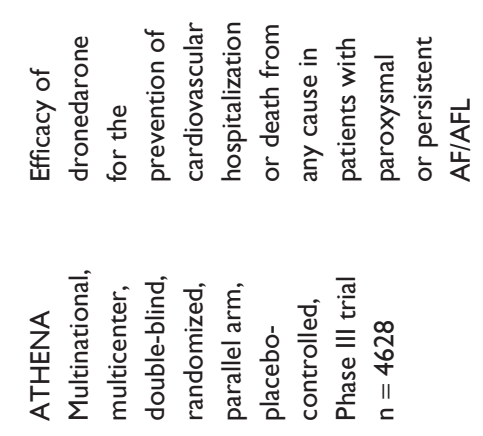



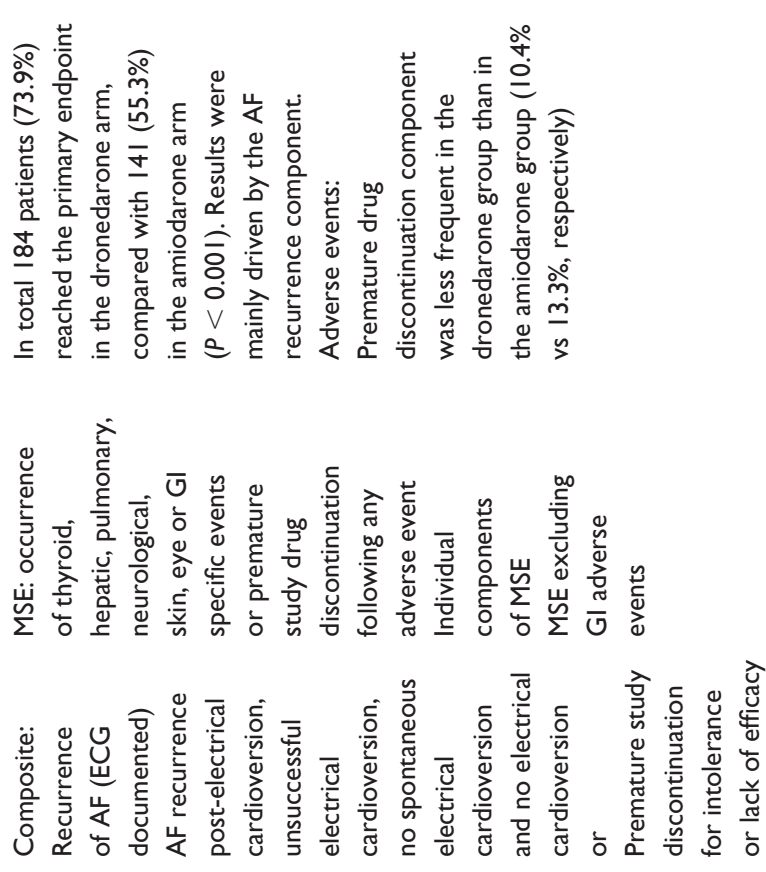

$\stackrel{\circ}{\xi}$

$\wedge$
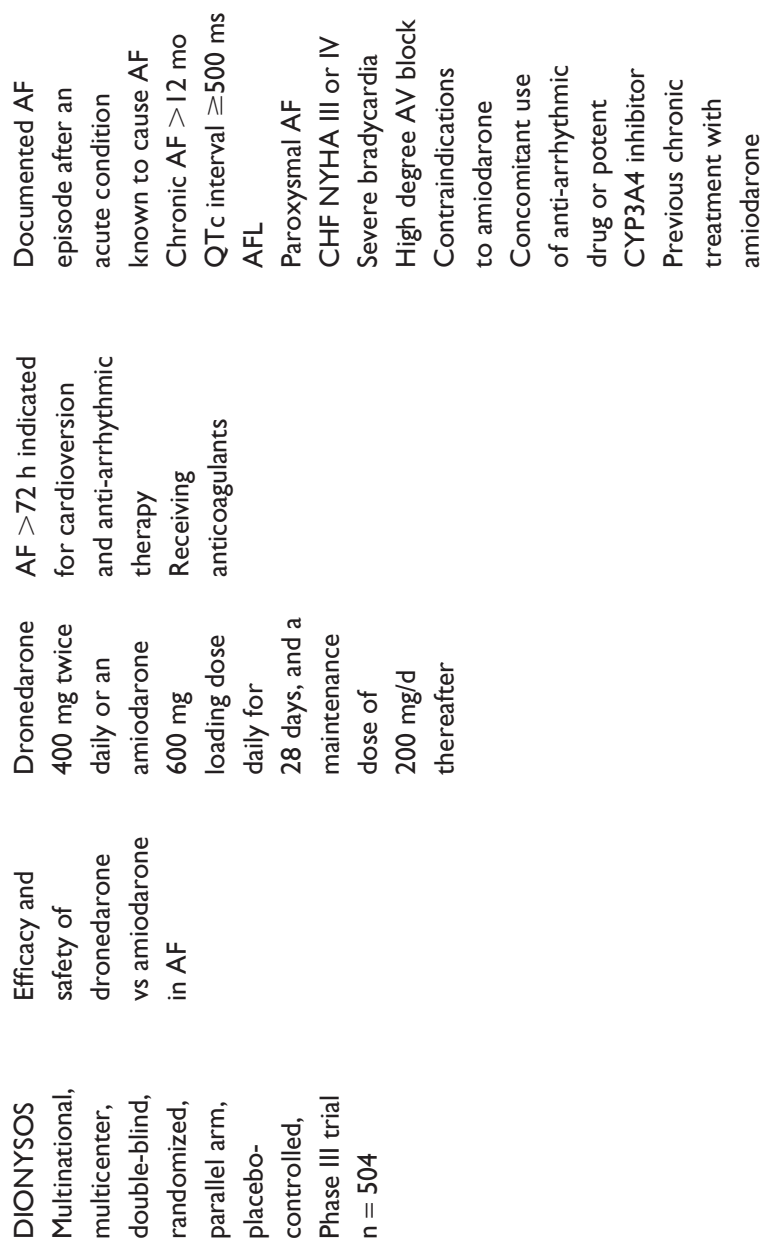

group $(P<0.001)$. Adverse event rate was similar between the drug and placebo. No TdP tachycardia occurred and there was no evidence of organ toxicity. However a slight increase in serum creatinine levels was observed in the dronedarone group, which returned to baseline when the drug was discontinued. Pooled post-hoc analysis of EURIDIS/ADONIS showed that dronedarone significantly reduced the relative risk of all-cause hospitalization or death by $27 \%$ compared with placebo $(P=0.01)$, which was further investigated in the ATHENA trial.

\section{ERATO $^{41}$}

The objective of ERATO (European Study of Dronedarone in Atrial Fibrillation) was to evaluate the efficacy of dronedarone for the control of mean 24-hour ventricular rate in patients with symptomatic, permanent AF, when added to concomitant therapy (ie, $\beta$-blockers [excluding sotalol], calcium-channel blockers, digoxin). The multicenter, double-blind, parallel-arm trial randomized 174 patients to receive either placebo or dronedarone $400 \mathrm{mg}$ twice a day for 6 months. The primary endpoint was the mean 24-hour ventricular rate after 2 weeks. Secondary endpoints included ventricular rate during submaximal and maximal exercise after 2 weeks, change in maximal exercise duration after 2 weeks, and mean 24-hour ventricular rate after 4 months.

Dronedarone decreased the 24-hour ventricular rate by $11.7 \mathrm{bpm}(P<0.0001)$ and the maximum exercise ventricular rate by $24.5 \mathrm{bpm}(P<0.0001)$, which was achieved on top of baseline medication including classic rate control drugs. There was no significant difference in exercise duration from baseline in either group, indicating that dronedarone benefits were achieved without impairing exercise capacity. This effect was sustained during long-term treatment ( 6 months). Serious adverse events were experienced by $17 \%$ of patients in the dronedarone group vs $14 \%$ in the placebo group, and the discontinuation rate was $15 \%$ in the dronedarone group vs $10 \%$ in the placebo group. These differences were nonsignificant. No evidence of organ toxicity or proarrhythmia was apparent in the dronedarone group. Frequent adverse events included infections ( $31 \%$ in the dronedarone group vs $25 \%$ in the placebo group) and gastrointestinal side effects (20\% in the dronedarone group vs $14 \%$ in the placebo group) and were not significantly different between the groups.

\section{ANDROMEDA $^{42}$}

ANDROMEDA (Antiarrhythmic Trial with Dronedarone in Moderate to Severe CHF Evaluating Morbidity Decrease) 
aimed at assessing potential benefit of dronedarone on all-cause mortality and hospitalization for worsening heart failure in high risk patients with congestive heart failure (NYHA III-IV or recent decompensation). Among study patients, $25 \%$ showed AF at randomization. The phase III trial design was multi-center, double-blind, parallel-arm and placebo-controlled. Patients were randomized into the dronedarone $400 \mathrm{mg}$ twice a day or placebo groups. These totaled 627 patients who were hospitalized with new or worsening heart failure, and who had experienced at least one episode of shortness of breath on minimal exertion or at rest, or who had paroxysmal nocturnal shortness of breath within the month before admission (NYHA III-IV), and who showed a wallmotion index at screening not exceeding 1.2 (left ventricular ejection fraction [LVEF] $35 \%$ or less). Primary endpoint was defined as death from any cause or hospitalization for worsening heart failure. Secondary endpoints included death from all causes, hospitalization for worsening heart failure, occurrence of AF/AFL, death from arrhythmia, or sudden death. The trial was prematurely stopped by the independent safety monitoring board after 7 months of first randomization because of an excess of deaths in the dronedarone arm. A total of 627 patients (310 in the dronedarone and 317 in the placebo group) had been enrolled up to the termination of the study. During a median follow-up of 2 months, 25 patients in the dronedarone group $(8.1 \%)$ and 12 patients in the placebo group $(3.8 \%)$ died (hazard ratio [HR] of dronedarone, 2.13; 95\% confidence interval [CI], 1.07-4.25; $P=0.03)$. The excess mortality was predominantly related to worsening heart failure, which accounted for 10 deaths in the dronedarone group compared with 2 in the placebo group. No case of death due to TdP tachycardia was reported. Subgroup analysis indicated that risk was increased in patients with a low wall-motion index. However, the most powerful predictor of death was treatment with dronedarone (HR, $2.19 ; 95 \%$ CI, $1.06-4.52 ; P=0.03)$. No statistical difference in the primary endpoint was detected between the two groups (HR, 1.38; 95\% CI, 0.92-2.09; $P=0.12$ ). Serious adverse events included more cases of increased creatinine concentration in the dronedarone group than in the placebo group. As a consequence of ANDROMEDA, patients with an unstable hemodynamic situation were excluded from the ATHENA trial.

\section{ATHENA ${ }^{43}$}

The study was designed to evaluate the efficacy and safety of dronedarone $400 \mathrm{mg}$ twice a day vs placebo for the prevention of cardiovascular hospitalization or death from any cause over a minimum treatment and follow-up duration of 12 months. Patients at risk with ECG-documentation of both AF/AFL and NSR within the last 6 months were included. The rationale was that AF is associated with several cardiovascular diseases that influence outcome. Therefore a combined primary endpoint of cardiovascular hospitalization or death was chosen to assess benefits of the drug. Secondary endpoints included death from any cause, cardiovascular death, and first hospitalization for cardiovascular reasons.

ATHENA is the largest single antiarrhythmic drug trial ever conducted. This multinational, multicenter, placebocontrolled, double-blind, parallel-arm randomized trial enrolled a total of 4628 patients; 2301 patients were randomly assigned to receive dronedarone and 2327 to receive placebo. Patients aged 75 years or older with or without additional risk factors were eligible. In addition, patients at least 70 years of age were enrolled when exhibiting one or more of the following risk factors: hypertension, diabetes, prior stroke/transient ischemic attack or systemic embolism, left atrial diameter $\geq 50 \mathrm{~mm}$, and LVEF $<40 \%$. Initially, the protocol had also allowed the inclusion of younger patients with additional risk factors. During the trial, overall mortality figures were lower than expected. Thus the steering committee had recommended changing the inclusion criteria to enrich the risk profile of the overall study population and patients younger than 70 years of age were no longer eligible. Patients included were of higher risk than the EURIDIS/ ADONIS study population. ${ }^{40,43}$ Exclusion criteria included permanent AF, congestive heart failure, NYHA class 4 within the last 4 weeks, and concomitant use of antiarrhythmic drugs or potent CYP3A4 inhibitors. Patients with NSR were directly randomized; AF/AFL was treated by cardioversion to achieve NSR before randomization. ${ }^{43}$ The mean follow-up period was $21 \pm 5$ months.

A primary outcome event occurred in 734 patients $(31.9 \%)$ in the dronedarone group and in 917 patients (39.4\%) in the placebo group (HR for dronedarone, 0.76; 95\% CI, 0.69-0.84; $P<0.001)$. Overall deaths from any cause occurred in 116 patients $(5.0 \%)$ in the dronedarone group and 139 patients $(6.0 \%)$ in the placebo group (HR for dronedarone, $0.84 ; 95 \% \mathrm{CI}, 0.66-1.08 ; P=0.18)$. Deaths from cardiovascular causes were lower in the dronedarone group (63 patients, 2.7\%) than in the placebo group (90 patients, $3.9 \%$; HR 0.71; 95\% CI, 0.519-0.98; $P=0.03$ ).

Adverse events occurred more often in the dronedarone group $(12.7 \%)$ than in the placebo group (8.1\%) and most frequently were of GI cause (26\% vs $22 \%)$. Bradycardia, 
QT- prolongation, diarrhea, nausea, rash, and increase in the serum creatinine level were significantly more common in the dronedarone group than in the placebo group. One single case of TdP tachycardia occurred in the dronedarone group. There were no significant differences in terms of organ toxicity, namely pulmonary symptoms, interstitial lung disease, and thyroid dysfunction.

Post hoc analysis of ATHENA data indicated that dronedarone decreases the risk of stroke in $\mathrm{AF} .{ }^{43}$ Dronedarone reduced the stroke rate from $1.8 \%$ per year to $1.2 \%$ per year (HR, 0.66; 95\% CI, 0.46-0.96, $P=0.027$ ). The effect of dronedarone was similar whether or not patients were receiving oral anticoagulant therapy, and there was a significantly greater effect of dronedarone in patients with higher $\mathrm{CHADS}_{2}$ scores (mean $\mathrm{CHADS}_{2}$ score was 2 in the ATHENA population). In addition dronedarone on top of standard therapy significantly reduced the relative risk of first cardiovascular hospitalization (HR, 0.74; $P<0.001){ }^{44,45}$ Furthermore, rhythm and rate control properties in ATHENA were objective of secondary analyses: the median time to first AF recurrence of patients in NSR at baseline was prolonged from 498 days in placebo patients to 737 days with dronedarone (HR, 0.75; $P<0.001)$ and dronedarone reduced the median heart rate in AF by $9 \mathrm{bpm}$ compared with placebo (75 vs 84 bpm; $P<0.001){ }^{46}$

\section{DIONYSOS ${ }^{47}$}

The DIONYSOS (Efficacy and Safety of Dronedarone Versus Amiodarone for the Maintenance of Sinus Rhythm in Patients With Atrial Fibrillation) trial investigated safety and efficacy of dronedarone compared with amiodarone for the maintenance of NSR in patients with persistent AF in a short-term study with a median treatment duration of 7 months. Inclusion criteria comprised patients with ECGdocumented AF for more than 72 hours for whom cardioversion and antiarrhythmic treatment was indicated. Exclusion criteria were AFL, contraindication to oral anticoagulation or to amiodarone, and paroxysmal AF. The trial was conducted in a multinational, multicenter, double-blind, parallel-arm, placebo-controlled fashion. Patients (504) were randomized either to a dronedarone $400 \mathrm{mg}$ twice a day group (249 patients) or to an amiodarone group (255 patients), receiving $600 \mathrm{mg}$ loading dose daily for 28 days, and a maintenance dose of $200 \mathrm{mg}$ daily thereafter. Electrical cardioversion was performed between days 10 and 28 if the patient had not converted to NSR spontaneously.

The composite primary endpoint was AF recurrence or premature study discontinuation for intolerance or lack of efficacy. The study also defined a main safety endpoint (MSE) of predefined thyroid, hepatic, pulmonary, neurological, skin, ocular and GI adverse events, as well as premature drug discontinuation due to any adverse event. Furthermore analysis of individual components of the MSE, and the MSE excluding GI adverse events, served as secondary endpoints.

The primary composite endpoint was in favor of amiodarone (HR, 1.59; 95\% CI, 1.28-1.98; $P<0.0001)$ and was driven mainly by the AF recurrence component, demonstrating that dronedarone was less effective than amiodarone in terms of prevention of AF recurrence (36.5\% vs $58.0 \%)$, while on the other hand, the premature drug discontinuation component was less frequent in the dronedarone group (10.4\% vs $13.3 \%$, respectively).

Analysis of the MSE suggested that dronedarone may have a more favorable safety profile. Incidence of MSE was $39.3 \%$ and $44.5 \%$ in the dronedarone and amiodarone groups, respectively at 12 months treatment (HR, 0.8; 95\% $\mathrm{CI}, 0.6-1.07 ; P=0.13)$. The reduced MSE in the dronedarone group was mainly due to fewer thyroid, neurological, skin and ophthalmologic events. However, more GI events (mainly diarrhea) were documented in the dronedarone group than in the amiodarone group (9.2 vs 3.1\% respectively of patients who had had least one episode of diarrhea). A prespecified endpoint focusing only on more clinically severe adverse events, excluding GI events, showed a statistically significant relative risk reduction in favor of dronedarone (HR, 0.61 ; 95\% CI, 0.44-0.84; $P=0.002$ ). Moreover, fewer patients receiving dronedarone developed bradycardia (defined as heart rate $<50 \mathrm{bpm}$ and decrease from baseline $>20 \mathrm{bpm}$ ) compared with amiodarone $(19.0 \%$ vs $29.5 \% ; P=0.0067)$ and QTc-interval (Bazett) prolongations $>500 \mathrm{~ms}$ were reported in $10.9 \%$ vs $20.5 \%$ of patients, respectively $(P=0.0033)$. The overall incidence of adverse events leading to permanent drug discontinuation was $12.9 \%$ in the dronedarone group compared with $17.6 \%$ in the amiodarone group and was mainly driven by QT-prolongation and hypoor hyperthyroidism.

Importantly there was an interaction between amiodarone and oral anticoagulants leading to supratherapeutic INR $(>4.5)$ levels associated with increased incidence of hemorrhagic events (number of bleeding events: 5.6\% in dronedarone and $11.4 \%$ in amiodarone groups; $P=0.03$ ). Moreover, dronedarone had an antihypertensive effect compared with amiodarone, which may contribute to beneficial effects on cardiovascular outcome observed in the ATHENA trial. There were no outcome data evaluated in 
the DIONYSOS trial. As a limitation of the study, authors stressed that short study-duration might have led to an underestimation of the adverse events in patients treated with amiodarone, since pulmonary events usually occur up to 2 years after initiation of amiodarone therapy. ${ }^{48}$

\section{Ongoing trials}

The PALLAS (Permanent Atrial fibrillation outcome Study using Dronedarone on top of standard therapy) trial started randomization in summer 2010, and is focused on patients with permanent AF and additional risk factors. It aims at prevention and reduction of cardiovascular outcome in these patients. The primary endpoint is defined as major cardiovascular events (stroke, systemic arterial embolism, myocardial infarction, or cardiovascular death) and first unplanned cardiovascular hospitalization or death from any cause.

The ARTEMIS AF (A Randomized, international, multicenter, open-label study to document optimal timing of initiation of dronedarone Treatment after conversion with loading dose of amiodarone in patients with persistent atrial fibrillation requiring conversion of Atrial Fibrillation) trial started randomization in summer 2010, and addresses the optimal regimen for dronedarone initiation following amiodarone discontinuation. It is divided into two sub-studies: the ARTEMIS AF loading trial starts at conversion with loading dose of amiodarone in patients with persistent AF requiring conversion of AF. The ARTEMIS AF long-term trial examines the initiation of dronedarone after long-term amiodarone in patients with paroxysmal or persistent AF, whatever the reason for change of treatment was.

\section{Concluding remarks}

The results of the ATHENA trial provided reassurance about the use of dronedarone. In particular, safety concerns raised by the results of the ANDROMEDA trial were addressed. In both trials patients with low LVEF and NYHA class II or III were included, but the ANDROMEDA population had been hospitalized for deterioration of heart failure, while stable outpatients were enrolled in the ATHENA trial. Further analysis performed by the manufacturer indicated why patients with LVEF $<35 \%$ and NYHA class III heart failure responded differently in the two trials. They concluded that clinical instability rather than LVEF or NYHA classification may be an important determinant of the treatment response to dronedarone. Because of lessons learned from ANDROMEDA, the FDA Cardiovascular and Renal Drugs Advisory
Committee prompted the drug to be contraindicated in heart failure NYHA classes III-IV or unstable NYHA class II. ${ }^{29}$ Owing to partially overlapping populations of ATHENA and ANDROMEDA, it appears premature to assume safety for the use in patients with advanced heart failure. Even if heart failure is in a stable chronic state, patients should be monitored for worsening of symptoms to discontinue dronedarone in case of deterioration. Further investigations need to identify more clearly populations taking advantage of, or being at risk during dronedarone therapy.

On the other hand, the DIONYSIS trial showed that dronedarone is less efficient than amiodarone in decreasing AF recurrence. At the same time, the trial indicated a more favorable side effect profile. In particular, dronedarone showed fewer thyroid, neurological, skin and ocular events, despite the fact that GI events were more frequent. For clinical practice the critical question is whether these potential benefits justify a retreat from the more efficient amiodarone. It is estimated that for every 1000 patients treated with dronedarone instead of amiodarone, there will be 228 more recurrences of AF per year in exchange for 9.6 fewer deaths and 62 fewer adverse events requiring drug discontinuation. This estimation is based on the assumption that indirect meta-analysis results are given the same approximate weight as the DIONYSOS direct comparison. ${ }^{49}$

There was no interaction between dronedarone and oral anticoagulants leading to a significantly reduced incidence of hemorrhagic events compared with amiodarone, an important issue for therapy of a disease that often requires oral anticoagulation. The transition from other antiarrhythmic drugs (including amiodarone to dronedarone and vice versa) requires special attention. In the ANDROMEDA and ATHENA trials, amiodarone was discontinued at least 4 weeks before randomization. Other class I or III drugs were stopped for at least 5 plasma half-lives before the first dose of dronedarone. Pending reliable data (ARTEMIS AF), one should adopt these recommendations for daily practice. Furthermore, transition from dronedarone to a different antiarrhythmic agent requires a drug-free interval of at least 5 plasma half-lives $(\sim 1$ week) after cessation of treatment with dronedarone.

Generally, one might ask what kind of benefit could be expected from another drug with less antiarrhythmic efficacy, but better tolerance than generically available amiodarone..$^{50,51}$ In this context, the ATHENA trial elucidated mortality/morbidity criteria beyond AF recurrence to address implications of dronedarone in cardiovascular 
disease associated with AF. Indeed, in addition to rhythm and rate control, ATHENA indicated outcome benefits of dronedarone in patients with $\mathrm{AF}$ and concomitant risk factors. Primary endpoint of the study was a combined endpoint of cardiovascular hospitalization or death, but the results were mainly driven by a lower hospitalization rate in the dronedarone $(29.3 \%)$ than in the placebo group (36.9\%), whereas deaths from any cause showed no significant difference. Further analysis indicated that death from cardiovascular cause was significantly lower in the dronedarone group than in placebo, but results have to be judged with caution. According to the FDA because of a pre-specified hierarchical procedure to control global type I error at the $5 \%$ level, cardiovascular death can only be tested if death from any cause is significant. ${ }^{29}$ Thus, further evidence with respect to total mortality will be needed in order to demonstrate clearly, hard endpoint benefits. In contrast, amiodarone did not improve cardiovascular morbidity or mortality. A recent meta-analysis in a large cohort of 12 studies revealed that while amiodarone was a potent antiarrhythmic drug, effective in achieving and maintaining NSR in persistent $\mathrm{AF}(P<0.00001$ vs placebo $)$, effects on mortality or hospitalization could not be shown. ${ }^{52}$

Moreover, dronedarone was effective in controlling ventricular rate after AF relapse as observed in EURIDIS/ ADONIS and achieved significant rate control in permanent AF as demonstrated by ERATO. Thus, dronedarone offers adequate treatment for rate control, and other than amiodarone, provides these effects without the price of intolerance and organ toxicity.

Based on the results of previous clinical trials, we conclude that dronedarone moderately reduces the incidence of AF relapses and offers an additional treatment option for rate control. Compared with amiodarone, dronedarone exhibits less efficacy but suggests better tolerance, pending long-term safety data. Furthermore, given that amiodarone is ineffective on cardiovascular hospitalization or death, ${ }^{52}$ dronedarone, beyond decreasing AF episodes, reduces cardiovascular hospitalizations and may reduce cardiovascular mortality. ${ }^{43}$ Notably, dronedarone is contraindicated in decompensating heart failure and NYHA classes III-IV. Considerations prior to initiating treatment with dronedarone in an individual patient should include the underlying disease (ie, heart failure), concomitant drugs (ie, oral anticoagulation, CYP3A4 inhibitors), basal heart rate, length of the QT interval, priority of maintaining NSR, and contraindications against amiodarone or class Ic agents.
In future studies, the antiarrhythmic potency of dronedarone should be directly compared with other antiarrhythmic drugs (eg, sotalol, class Ic agents) to evaluate efficacy and safety. Furthermore, questions remain regarding long-term safety benefits (organ toxicity) compared with amiodarone, as DIONYSOS was conducted as a short-term trial only. Future studies will have to clarify the drug's impact on overall mortality, particularly in patients with heart failure. ATHENA and ANDROMEDA raised questions concerning the outcome in patients with moderate to severe congestive heart failure at risk of further deterioration. Moreover, potential benefits for the reduction of stroke risk have to be studied using prespecified endpoints, as current data from ATHENA subanalysis ${ }^{43}$ indicating reduction of stroke risk by dronedarone were obtained only by post-hoc analysis.

In addition, the benefit of dronedarone in patients after $\mathrm{AF}$ recurrence compared with classic rate control agents would be of interest. In particular, data on rate control properties of dronedarone independent from underlying baseline medication are lacking, preventing comparison with standard rate control approaches. ${ }^{29}$ Finally, the application of dronedarone in the management of additional arrhythmias (ie, ventricular tachycardia) may be evaluated in the near future.

\section{Acknowledgment}

This work was supported in part by grants from the MaxPlanck-Society (TANDEM project to PAS) and from the German Heart Foundation/German Foundation for Heart Research (to DT).

\section{Disclosure}

The authors declare that they have no conflict of interest.

\section{References}

1. Benjamin EJ, Wolf PA, D'Agostino RB, Silbershatz H, Kannel WB, Levy D. Impact of atrial fibrillation on the risk of death: the Framingham Heart Study. Circulation. 1998;98(10):946-952.

2. Dulli DA, Stanko H, Levine RL. Atrial fibrillation is associated with severe acute ischemic stroke. Neuroepidemiology. 2002;2(3-4):118-123.

3. Wang TJ, Larson MG, Levy D, Vasan RS, et al. Temporal relations of atrial fibrillation and congestive heart failure and their joint influence on mortality: the Framingham Heart Study. Circulation. 2003; 107(7):2920-2925.

4. Wolf PA, Abbott RD, Kannel WB. Atrial fibrillation as an independent risk factor for stroke: the Framingham Study. Stroke. 1991;22(8): 983-988.

5. Wyse DG, Slee A, Epstein AE, et al. Alternative endpoints for mortality in studies of patients with atrial fibrillation: the AFFIRM study experience. Heart Rhythm. 2004;5(11):531-537.

6. Stewart S, Hart CL, Hole DJ, McMurray JJ. A population-based study of the long-term risks associated with atrial fibrillation: 20-year follow-up of the Renfrew/Paisley study. Am J Med. 2002;113(5): 359-364. 
7. Fuster V, Rydén LE, Cannom DS, et al. ACC/AHA/ESC 2006 guidelines for the management of patients with atrial fibrillation. Europace. 2006;8(9):651-745.

8. Fuster V, Rydén LE, Cannom DS, et al. ACC/AHA/ESC 2006 guidelines for the management of patients with atrial fibrillation: Executive summary: A report of the American College of Cardiology/ American Heart Association Task Force on Practice Guidelines and the European Society of Cardiology Committee for Practice Guidelines and Policy Conferences. J Am Coll Cardiol. 2006;48(8):854-906.

9. Go AS, Hylek EM, Phillips KA, et al. Prevalence of diagnosed atrial fibrillation in adults: national implications for rhythm management and stroke prevention: the AnTicoagulation and Risk Factors in Atrial Fibrillation (ATRIA) Study. JAMA. 2001;285(5):2370-2375.

10. Camm JA, Reiffel JA. Defining endpoints in clinical trials on atrial fibrillation. Eur Heart J Suppl. 2008;10(Suppl H):H55-H78.

11. Naccarelli GV, Varker H, Lin J, Schulman KL. Increasing prevalence of atrial fibrillation and flutter in the United States. Am J Cardiol. 2009; 104(12):1534-1539.

12. Nattel S, Carlsson L. Innovative approaches to anti-arrhythmic drug therapy. Nat Rev Drug Discov. 2006;5(12):1034-1049.

13. Nattel S, Opie LH. Controversies in atrial fibrillation. Lancet. 2006; 367(9506):262-272.

14. Nattel S. New ideas about atrial fibrillation 50 years on. Nature. 2002; 415(6868):219-226.

15. Nattel S, Burstein B, Dobrev D. Atrial remodeling and atrial fibrillation: mechanisms and implications. Circ Arrhythmia Electrophysiol. 2008;1(1):62-73.

16. Allessie MA, Bonke FI, Schopman FJ. Circus movement in rabbit atrial muscle as a mechanism of tachycardia III. The "leading circle" concept: a new model of circus movement in cardiac tissue without the involvement of an anatomical obstacle. Circ Res. 1977;41(1):9-18.

17. Comtois P, Kneller J, Nattel S. Of circles and spirals: bridging the gap between the leading circle and spiral wave concepts of cardiac reentry. Europace. 2005;7(suppl 2):10-20.

18. Davidenko JM, Pertsov AV, Salomonsz R, Baxter W, Jalife J. Stationary and drifting spiral waves of excitation in isolated cardiac muscle. Nature. 1992;355(6358):349-351.

19. Pertsov AM, Davidenko JM, Salomonsz R, Baxter WT, Jalife J. Spiral waves of excitation underlie reentrant activity in isolated cardiac muscle. Circ Res. 1993;72(3):631-650.

20. Dobrev D, Nattel S. New antiarrhythmic drugs for treatment of atrial fibrillation. Lancet. 2010;375(9721):1212-1223.

21. Hart RG, Pearce LA, Aguilar MI. Meta-analysis: antithrombotic therapy to prevent stroke in patients who have non-valvular atrial fibrillation. Ann Intern Med. 2007;146(12):857-867.

22. Carlsson J, Miketic S, Windeler J, et al. Randomized trial of rate-control versus rhythm-control in persistent atrial fibrillation: the Strategies of Treatment of Atrial Fibrillation (STAF) study. J Am Coll Cardiol. 2003; 41(5):1690-1696.

23. Hohnloser SH, Kuck KH, Lilienthal J. Rhythm or rate control in atrial fibrillation-Pharmacological Intervention in Atrial Fibrillation (PIAF): a randomized trial. Lancet. 2000;356(11):1789-1794.

24. Van Gelder IC, Hagens VE, Bosker HA, et al. A comparison of rate control and rhythm control in patients with recurrent persistent atrial fibrillation. N Engl J Med. 2002;347(11):1834-1840.

25. Wyse DG, Waldo AL, DiMarco JP, et al. Atrial Fibrillation Follow-up Investigation of Rhythm Management (AFFIRM) Investigators. A comparison of rate control and rhythm control in patients with atrial fibrillation. N Engl J Med. 2002;347(12):1825-1833.

26. Echt DS, Liebson PR, Mitchell LB, et al. Mortality and morbidity in patients receiving encainide, flecainide, or placebo. The Cardiac Arrhythmia Suppression Trial. N Engl J Med. 1991;324(3): 781-788.

27. Camm AJ, Kirchhof P, Lip GY, et al. Guidelines for the management of atrial fibrillation: the Task Force for the Management of Atrial Fibrillation of the European Society of Cardiology (ESC). Eur Heart J. 2010;31(19):2369-2429.
28. Ehrlich JR, Nattel S, Hohnloser SH. Novel anti-arrhythmic drugs for atrial fibrillation management. Curr Vasc Pharmacol. 2007;5(3): $185-195$.

29. Oyetayo OO, Rogers CE, Hofmann PO. Dronedarone: a new antiarrhythmic agent. Pharmacotherapy. 2010;30(9):904-915.

30. Kathöfer S, Thomas D, Karle CA. The novel antiarrhythmic druc dronedarone: comparison with amiodarone. Cardiovasc Drug Rev. 2005;23(3):217-230.

31. Patel PD, Bhuriya R, Patel DD, Arora BL, Singh PP, Arora RR. Dronedarone for atrial fibrillation: a new therapeutic agent. Vasc Health Risk Manag. 2009;5:635-642.

32. Sun W, Sarma JS, Singh BN. Chronic and acute effects of dronedarone on the action potential of rabbit atrial muscle preparations: comparison with amiodarone. J Cardiovasc Pharmacol. 2002;39(5):677-684.

33. Thomas D, Kathofer S, Zhang W, et al. Acute effects of dronedarone on both components of the cardiac delayed rectifier $\mathrm{K}^{+}$current, HERG and KvLQT1/minK potassium channels. Br J Pharmacol. 2003; 140(5):996-1002.

34. Gautier P, Guillemare E, Marion A, Bertrand JP, Tourneur Y, Nisato D. Electrophysiologic characterization of dronedarone in guinea pig ventricular cells. J Cardiovasc Pharmacol. 2003;41(2):191-202.

35. Hodeige D, Heyndrickx JP, Chatelain P, Manning A. SR 33589, a new amiodarone-like antiarrhythmic agent: anti-adrenoceptor activity in anaesthetized and conscious dogs. Eur J Pharmacol. 1995;279(6): $25-32$.

36. Guiraudou P, Pucheu SC, Gayraud R, et al. Involvement of nitric oxide in amiodarone- and dronedarone-induced coronary vasodilation in guinea pig heart. Eur J Pharmacol. 2004;496(8):119-127.

37. Touboul P, Brugada J, Capucci A, Crijns HJ, Edvardsson N, Hohnloser SH. Dronedarone for prevention of atrial fibrillation: a dose-ranging study. Eur Heart J. 2003;24(8):1481-1487.

38. Pollak PT, Shafer SL. Use of population modeling to define rational monitoring of amiodarone hepatic effects. Clin Pharmacol Ther. 2004; 75(4):342-351.

39. Tschuppert Y, Buclin T, Rothuizen LE, et al. Effect of dronedarone on renal function in healthy subjects. Br J Clin Pharmacol. 2007;64(12): 785-791.

40. Singh BN, Connolly SJ, Crijns HJ, et al. Dronedarone for maintenance of sinus rhythm in atrial fibrillation or flutter. N Engl J Med. 2007;357(9): 987-999.

41. Davy JM, Herold M, Hoglund C, et al; ERATO Study Investigators. Dronedarone for the control of ventricular rate in permanent atrial fibrillation: the Efficacy and safety of dRonedArone for the cOntrol of ventricular rate during atrial fibrillation (ERATO) study. Am Heart $J$. 2008;156(9):527.e1-e9.

42. Køber L, Torp-Pedersen C, McMurray JJ, et al. Increased mortality after dronedarone therapy for severe heart failure. N Engl J Med. 2008; 358(6):2678-2687.

43. Hohnloser SH, Crijns HJ, van Eickels M, et al. Effect of dronedarone on cardiovascular events in atrial fibrillation. $N$ Engl J Med. 2009; 360(2):668-678.

44. Connolly SJ, Crijns HJ, Torp-Pedersen C, et al. Analysis of stroke in ATHENA: a placebo-controlled, double-blind, parallel-arm trial to assess the efficacy of dronedarone $400 \mathrm{mg}$ BID for the prevention of cardiovascular hospitalization or death from any cause in patients with atrial fibrillation/atrial flutter. Circulation. 2009;120(9):1174-1180.

45. Torp-Pedersen C, Page RL, Connolly S, et al. The effect of dronedarone on hospitalizations in patients with atrial fibrillation. Results from the ATHENA study. Circulation. 2008;118(8):828.

46. Page RL, Connolly S, Crijns H, et al. Rhythm- and rate-controlling of dronedarone in patients with atrial fibrillation. Results from the ATHENA trial. Circulation. 2008;118(8):827.

47. Le Heuzey JY, De Ferrari GM, Radzik D, Santini M, Zhu J, Davy JM. A short-term, randomized, double-blind, parallel-group study to evaluate the efficacy and safety of dronedarone versus amiodarone in patients with persistent atrial fibrillation: the DIONYSOS study. J Cardiovasc Electrophysiol. 2010;21(6):597-605. 
48. Bongard V, Marc D, Philippe V, Jean-Louis M, Maryse LM. Incidence rate of adverse drug reactions during long-term follow-up of patients newly treated with amiodarone. Am J Ther. 2006;13(7-8):315-319.

49. Piccini JP, Hasselblad V, Peterson ED, Washam JB, Califf RM, Kong DF. Comparative efficacy of dronedarone and amiodarone for the maintenance of sinus rhythm in patients with atrial fibrillation. $\mathrm{JAm}$ Coll Cardiol. 2009;54(12):1089-1095.

50. Hughes PJ, Freeman MK, Cohenour FV, Price EM. Dronedarone: an alternative to amiodarone? Consult Pharm. 2010;25(9):555-569.
51. Wolbrette D, Gonzalez M, Samii S, Banchs J, Penny-Peterson E, Naccarelli G. Dronedarone for the treatment of atrial fibrillation and atrial flutter: approval and efficacy. Vasc Health Risk Manag. 2010;6: 517-523.

52. Doyle JF, Ho KM. Benefits and risks of long-term amiodarone therapy for persistent atrial fibrillation: a meta-analysis. Mayo Clin Proc. 2009; 84(3):234-242.

\section{Publish your work in this journal}

Drug Design, Development and Therapy is an international, peerreviewed open-access journal that spans the spectrum of drug design and development through to clinical applications. Clinical outcomes, patient safety, and programs for the development and effective, safe, and sustained use of medicines are a feature of the journal, which has also been accepted for indexing on PubMed Central. The manuscript management system is completely online and includes a very quick and fair peer-review system, which is all easy to use. Visit http://www.dovepress.com/testimonials.php to read real quotes from published authors.

Submit your manuscript here: http://www.dovepress.com/drug-design-development-and-therapy-journal 